CALEI-

DOSCOPIO

$35 / 36$

खु

JULIO - DICIEMBRE 2016

ENERO - JUNIO 2017

\title{
Prensa y revolución en Monterrey: el surgimiento del diario El Porvenir (1919-1922)
}

FELIPE BÁRCENAS GARCÍA ${ }^{1}$

\section{RESUMEN}

El artículo analiza el papel desempeñado por el diario El Porvenir, en Monterrey, Nuevo León, durante 1919-1922. En este periodo, el artículo 123 de la Constitución de 1917 propició un brote sin precedentes de movilizaciones obreras. Mientras que los trabajadores exigían la aplicación inmediata y plena de sus nuevos derechos constitucionales (jornadas laborales de ocho horas, descanso dominical y seguridad en las áreas de trabajo, entre otros), los empresarios se negaban a modificar sus esquemas administrativos. En este contexto, El Porvenir emergió como un medio que, comprometido con los hombres de negocios, intentó contrarrestar el activismo laboral.

Palabras clave: El Porvenir, Monterrey, prensa, revolución, Nuevo León.

\section{ABSTRACT}

This article analyzes the role played by the newspaper El Porvenir in Monterrey (Nuevo León) during 1919-1922. In this period, the article 123

1 Candidato a doctor en Humanidades por parte de la UAM-I. Maestro en Historia Moderna y Contemporánea por el Instituto Mora. 
of the 1917's Constitution caused an unprecedented outbreak of labor mobilizations. While workers demanded immediate and total implementation of its new constitutional rights (working days of eight hours, Sunday rest and security in working areas), employers refused to change the administrative schemes. In this context, El Porvenir emerged as a medium which supported businessmen and sought to counter the labor activism.

Keywords: El Porvenir, Monterrey, press, revolution, Nuevo León.

\section{INTRODUCCIÓN}

En nenero de 1919, el poeta colombiano Ricardo Arenales (mejor conocido como Porfirio Barba Jacob) fundó en Monterrey el diario El Provenir. Éste fue uno de los periódicos nuevoleoneses contemporáneos más relevantes, debido al poder de divulgación que consiguió y a la relación que sus propietarios y redactores mantuvieron con la élite empresarial regiomontana, razones por las cuales jugó un papel político relevante en diversos momentos del siglo XX. ${ }^{2}$ Una prueba de su trascendencia radica en el hecho de que, a diferencia de lo efímeras que fueron las publicaciones impresas tras el estallido de la Revolución mexicana, el diario de Arenales es el único rotativo de Nuevo León establecido durante los diez años que ha perdurado hasta la actualidad.

En este artículo se analizan los factores que suscitaron el surgimiento de El Porvenir, así como la función que desempeñó en Monterrey durante 1919-1922, un periodo en el cual se debatió fuertemente el establecimiento de una legislación laboral en Nuevo León (luego de promulgarse la Constitución de 1917). En este contexto, es primordial estudiar El Porvenir para entender el desarrollo de la prensa regiomontana a lo largo del siglo xx, ya que, al ser considerado durante décadas como el diario más importante del estado, marcó las pautas del ejercicio periodístico local.

2 Por élite empresarial regiomontana aludimos a ese "espectro de grupos familiares que dirigieron el desarrollo capitalista de Nuevo León" a partir de 1890, compuesto principalmente por los apellidos: Armendáriz, Calderón, Ferrara, Garza, HernándezMendirichaga, Madero, Milmo, Muguerza, Rivero, Sada y Zambrano. Cerutti, Mario, "Producción capitalista y articulación del empresariado en Monterrey (1890-1910)", en Labastida, Julio (comp.), Grupos económicos y organizaciones empresariales en México, México, Biblioteca Iberoamericana-Alianza Editorial Mexicana-Universidad Nacional Autónoma de México, 1986, pp. 17 y 25. 
Se partirá de dos supuestos: 1) que El Porvenir emergió como un diario comprometido con los intereses de los prominentes empresarios de Monterrey; y 2) que El Porvenir fue el primer proyecto instituido para contrarrestar sistemáticamente la influencia que la Revolución ejerció en Nuevo León.

El objetivo de la investigación se resolverá en tres apartados. En los primeros dos se explica cómo El Porvenir fue fundado para combatir el activismo obrero de Monterrey durante la década de 1910. En el tercero, se examina la estrategia que diseñó el diario de Arenales para defender los intereses empresariales.

\section{REVOLUCIÓN Y MOVIMIENTO OBRERO EN MONTERREY}

A principios del siglo $\mathrm{xx}$, periodistas y viajeros ensalzaban con entusiasmo el progreso industrial de Nuevo León, jactándose de la armonía entre clases que prevalecía al interior de las fábricas. No obstante, esta visión fue criticada fuertemente a partir de 1910, cuando la Revolución expuso las problemáticas sociales existentes en el estado: tras el derrocamiento de Porfirio Díaz, emergió un activismo obrero que cuestionó los esquemas administrativos de las más reconocidas industrias locales, planteó la formación de sindicatos como un derecho y organizó huelgas para demandar el mejoramiento de las condiciones tanto económicas como laborales.

Si bien las luchas armadas no ocasionaron grandes daños materiales ni un número elevado de muertes en Monterrey, las repercusiones sociales y políticas que la Revolución provocó fueron significativas. De acuerdo con Abraham Nuncio,

[... que la Revolución no haya adquirido la intensidad destructiva de otros estados, se debió en parte a la condición industrial de la ciudad. Pero esta tenía que presentarse de algún modo. En Monterrey había fuerzas sociales exacerbadas, las cuales se desataron al estallar la revuelta en $1910 .^{3}$

La fuerza social que más se desató en la capital nuevoleonesa durante la Revolución fue el sector obrero, el cual aprovechó la coyun-

3 Nuncio, Abraham, Visión de Monterrey, México, Fondo de Cultura Económica, 2006, p. 106. 
tura bélica para exponer públicamente una serie de demandas. Cabe señalar que durante el régimen de Bernardo Reyes (1885-1909) ya se habían presentado algunos brotes de inconformidad laboral, pero fueron escasos y rápidamente controlados.

Los conflictos armados que acontecieron en Nuevo León provocaron carestía y desempleo. Los trabajadores respondieron a estas condiciones mediante protestas, cuyos motivos, inicialmente, no iban más allá de la resolución inmediata a las crisis que los afectaban. ${ }^{4}$ Pero en el curso de la década, activistas laborales fueron más allá de la esfera de lo económico y organizaron un movimiento que exigió el reconocimiento legal de reivindicaciones relativas al salario, las condiciones de trabajo y la jornada laboral.

Se puede decir que la Revolución provocó que los obreros se convirtieran en una fuerza social y en una de las amenazas más grandes para la élite empresarial de Monterrey. Basta con mencionar que entre 1910 y 1920 tuvo lugar una inusitada oleada huelguística que involucró a la mayoría de las empresas más relevantes de la ciudad, entre ellas, la Cervecería Cuauhtémoc y las industrias del acero. ${ }^{5}$

Tras la promulgación de la Constitución de 1917, los obreros regiomontanos utilizaron el artículo 123 como un sustento legal para reclamar al gobierno la efectividad plena de sus nuevos derechos: jornadas laborales de ocho horas ( 7 horas para el turno nocturno), descanso dominical y seguridad e higiene en las áreas de trabajo, entre otros. Sin embargo, la carta magna otorgaba a cada estado la posibilidad de decretar un código laboral con base en sus condiciones locales. Por ello, entre 1917 y 1922 (cuando entró en vigor la primera ley del trabajo en Nuevo León), Monterrey fue el escenario de una fuerte pugna política en la que se enfrentaron trabajadores organizados que exigían el establecimiento inmediato de un código laboral local, contra un sector empresarial que se negaba a admitir cualquier ley que representara una intromisión a sus esquemas administrativos. ${ }^{6}$

4 Snodgrass, Michael, Deferencia y desafío en Monterrey. Trabajadores, paternalismo y Revolución en México, 1890-1950, Monterrey, Fondo Editorial de Nuevo León, 2008, pp. 48 y 54.

5 Rojas Sandoval, Javier, Movimiento obrero y partidos políticos en Nuevo León. 1910-1920, Monterrey, OIDMO, A. C., 1982, pp. 8-9.

6 Rojas Sandoval, Javier, Monterrey: poder político, obreros y empresarios en la coyuntura revolucionaria, Monterrey, Facultad de Filosofía y Letras, Universidad Autónoma de Nuevo León, 1992, pp. 17-21. 
¿De qué medios dispusieron los empresarios para ganar la batalla en torno a la aplicabilidad del código laboral? ¿Con qué aliados contaron para frenar el avance del movimiento obrero? Al no contar con un partidario fuerte en el gobierno que defendiera sus intereses, como en su momento lo fue Bernardo Reyes, los prominentes hombres de negocios se valieron de diversos recursos para contrarrestar por sí mismos la influencia de los trabajadores organizados, uno de ellos fue El Porvenir. La instauración de este diario marcó el inicio del periodismo empresarial contemporáneo de Monterrey. Dicho tipo de periodismo se caracterizó por dos elementos: 1) la búsqueda de ganancias económicas a través de la publicidad y la difusión de contenidos informativos; a diferencia de las publicaciones decimonónicas de opinión, cuya función era discutir ideas políticas mediante textos reflexivos diseñados para un público específico, los periódicos empresariales brindaron todo tipo de temas comerciales y sensacionalistas que pudiesen acaparar la atención de la mayor cantidad posible de lectores. 2) Su desarrollo implicó el establecimiento de una industria equipada con tecnología especializada para producir ejemplares a gran escala. ${ }^{7}$

\section{LA FUNDACIÓN DE EL PORVENIR}

Como ya se mencionó anteriormente, el poeta y periodista entonces conocido como Ricardo Arenales fundó El Porvenir en enero de 1919. Su nombre de pila era Miguel Ángel Osorio Benítez, pero en diversos momentos se hizo llamar Juan Sin Miedo, Juan Azteca, Juan Sin Tierra, Almafuerte, Junius, Cálifax, Maín Ximénez y Porfirio Barba Jacob. Nació el 29 de julio de 1883 en Santa Rosa de Osos, un pequeño pueblo bajo la jurisdicción de Antioquia, Colombia. ${ }^{8}$

De origen humilde, Osorio Benítez cursó apenas dos años de primaria, abandonó sus estudios para trabajar en el campo hasta los quince años de edad, cuando fue reclutado por el ejército colombiano. Según Elías Nandino, amigo del poeta, éste tuvo sus primeros contactos con los libros en condición de soldado:

7 Burkholder de la Rosa, Arno, "El periódico que llegó a la vida nacional. Los primeros años del diario Excélsior (1916-1932)", Historia Mexicana, México, El Colegio de México, vol. LVIII, núm. 4, abril-junio de 2009, pp. 1371-1381.

8 Rousset Banda, Guillermo (coord.), Porfirio Barba Jacob, obra poética, México, Editorial Domés, 1985, p. VIII. 
Entre la tropa había algunos que gustaban de las letras, comenzó a leer libros de versos y novelas [...]. Al dejar la soldadesca [...] consiguió ser maestro rural en alguna hacienda [... ]. Juntó un poco de plata en su chamba escolar y un día se lanzó a vagar por Centroamérica. Conoció poetas, discutió sobre poesía haciendo uso de su intuición y, sin saber cómo, después de leer a los clásicos, La Divina Comedia, El Quijote y los libros de los poetas que iba conociendo, hizo su debut en revistas literarias, en suplementos dominicales de los diarios. Y sin querer y sin saber fue sembrando poemas, inventado artículos en la prensa. ${ }^{9}$

Con una modesta experiencia en el periodismo literario adquirida en Centroamérica, con motivos poco claros y bajo el pseudónimo de Ricardo Arenales, Osorio Benítez llegó a Monterrey en 1908, un año en el que proliferaban las publicaciones literarias. Su talento y aptitudes para socializar le permitieron incursionar inmediatamente en el medio periodístico, escribiendo para El Espectador, diario afín a Bernardo Reyes. ${ }^{10}$ Sin embargo, su primera estancia en la capital nuevoleonesa fue corta: ese mismo año decidió emigrar a la Ciudad de México, donde escribió para la revista de orientación porfirista El Mundo Ilustrado. ${ }^{11}$

Tras el estallido de la Revolución mexicana, Arenales se propuso enfrentar la insurgencia con su pluma, para ello ingresó a la redacción de El Imparcial, periódico capitalino que en sus primeros años de vida asumió una postura reaccionaria. Posteriormente, cuando el general Victoriano Huerta derrocó a Francisco I. Madero, el colombiano defendió al régimen golpista a través de El Independiente. ${ }^{12}$

Es evidente que Arenales sentía antipatía hacia la Revolución, postura que le valió amenazas de muerte. Por ello, cuando Huerta fue derrotado, se vio forzado a exiliarse en Centroamérica y Cuba. Regresó a México hasta 1918, cuando la guerra entre constitucionalistas y convencionalistas había finalizado. Sorprendentemente, el poeta colombiano regresó con una actitud afín al gobierno revolucionario.

Es complicado determinar si el cambio en las opiniones políticas de Arenales fue la manifestación de una auténtica simpatía hacia el

9 Ibid., p. IX.

10 Cavazos Garza, Israel, Escritores de Nuevo León, diccionario biobibliográfico, Monterrey, Universidad Autónoma de Nuevo León, 1996, p. 266.

11 García Aguilar, Eduardo (investigación, selección y prólogo), Porfirio Barba Jacob. Escritos mexicanos, Bogotá, Fondo de Cultura Económica, 2009, p. 7.

12 Ibid., p. 8. 
nuevo Estado, o del oportunismo adoptado por intereses personales. Lo cierto es que a lo largo de su vida siempre expresó admiración por Porfirio Díaz y Bernardo Reyes. Con esta postura encontrada -respaldando a un gobierno derivado de una Revolución que criticó durante años, pero con un profundo respeto hacia el régimen porfirista-, Ricardo Arenales instauró El Porvenir.

Entre los miembros fundadores del periódico destacaron el impresor Jesús Cantú Leal (quien se convertiría en el propietario del diario a tan sólo unos meses de su establecimiento), Eduardo Martínez Celis (mismo que fungió como jefe de redacción), el editorialista David Alberto Cosío y el escritor Federico Gómez. Todos ellos se caracterizaron por su simpatía hacia las grandes industrias regiomontanas y sus propietarios. De ahí que El Porvenir ensalzara constantemente al sector empresarial, buscando mostrar "su importancia en la vida económica y en el desarrollo moral e intelectual de la sociedad". De acuerdo con el rotativo:

Uno de los propósitos que determinaron la fundación de este periódico, fue el de prestar un estímulo a todas las $[\ldots \mid$ empresas grandes y bien regidas $[\ldots \mid$. Tales empresas no sólo ofrecen trabajo a numerosos individuos $[\ldots]$ sino que constituyen verdaderas escuelas de acción inteligente y honesta, donde se templa el carácter, se aguzan las facultades, se aprende prácticamente la distribución de las faenas, la eficacia, el ahorro, la previsión, etc. ${ }^{13}$

Este hecho explica el posicionamiento que El Porvenir asumió ante los conflictos obrero-patronales generados a raíz de la Revolución. Aunque el diario de Arenales fue presentado como un periódico de tipo informativo (cuyo rasgo principal en la época era la pretensión de objetividad), no ocultó su compromiso con los influyentes empresarios de Monterrey. En la primera plana del ejemplar inaugural expresó que su empresa fue iniciada:

en pro de los grandes intereses locales, con la inserción de las opiniones que $[\ldots]$ se han servido dar exclusivamente para nuestro periódico, algunos de los caballeros que más alta posición ocupan en la vida de los negocios, y que mayor prestigio tienen por su honradez y su cultura. ${ }^{14}$

13 El Porvenir, 16 de abril de 1920.

14 El Porvenir, 31 de enero de 1919. 
Tales caballeros de los negocios que opinaron en las páginas de El Porvenir tenían por lo menos dos preocupaciones: detener la oleada huelguística y la fiebre constitucionalista provocadas por las mareas revolucionarias; y evitar -hasta donde fuese posible- la intervención del Estado en los asuntos empresariales. Es comprensible que en la sección editorial del mismo número inaugural se mencionara que otro de los propósitos de El Porvenir era que las clases obreras regresaran "hacia la perfección moral" que habían perdido tras el estallido de la Revolución. En otras palabras, el diario de Arenales buscó promover una conducta de total obediencia y lealtad entre los trabajadores, que no criticara bajo ninguna circunstancia los valores fomentados por los patrones. Para ello, se incluyó una columna dedicada a los obreros, de modo que, por primera vez en la historia de Monterrey, un periódico de circulación cotidiana se interesaba en generar un público lector compuesto por trabajadores urbanos. Desde luego que tal interés tenía motivos ideológicos.

Si bien, el empresariado hegemónico de Monterrey no fundó El Porvenir, lo empleó como su emisario desde la publicación del primer ejemplar. Por ello, no debe sorprendernos el éxito que la empresa periodística de Arenales consiguió: para 1922 (a tan sólo tres años de su fundación), El Porvenir ya era considerado por el gobierno estatal como el diario más importante y de mayor circulación en Nuevo León. ${ }^{15}$

Es pertinente señalar que uno de los inversionistas del diario fue el entonces gobernador carrancista Nicéforo Zambrano (1917-1919). ${ }^{16}$ Este hecho ayuda a explicar cómo la convergencia de grandes intereses económicos y políticos le permitió a El Porvenir posicionarse rápidamente como el medio informativo más relevante del estado.

Ahora bien, si el movimiento constitucionalista que encabezó Venustiano Carranza se apoyó inicialmente en los obreros, ¿por qué Zambrano invirtió recursos en un periódico que intentaba combatir el activismo laboral? Aludir únicamente al interés económico supondría responder parcialmente la cuestión. Hay que recordar que, tras la represión de la primera huelga general en México en 1916, Carranza limitó severamente las peticiones obreras que él mismo había defendido, a tal grado que decretó pena de muerte no sólo para los huelguistas,

15 Archivo Histórico de Nuevo León, Fondo Ministerio de Agricultura, Correspondencia, 1910-1928. Caja 1.

16 El Porvenir, 12 de febrero de 1919. 
sino también para quienes simpatizaran con ellos, pues arguyó que la reconstrucción económica del devastado país requería la reactivación inmediata de las actividades industriales.

El gobierno de Zambrano y la élite empresarial de Monterrey tenían un enemigo en común: el activismo laboral. Para frenar su avance prepararon una ofensiva mediática a través de El Porvenir, que consistió en presentar a Monterrey como un escenario en el que hombres rusos, sin nombres ni rostros, se encontraban secretamente dirigiendo las movilizaciones laborales.

\section{LA OFENSIVA DE EL PORVENIR}

Con el objetivo de moldear el imaginario social de los regiomontanos acorde a las ideas del sector empresarial, El Porvenir diseñó un plan centrado en un punto: atacar el socialismo y el comunismo, modelos tachados como la antítesis de la civilización, cuya influencia -se consideró desde el primer número- era la causante de la radicalización de los obreros.

Para el diario de Arenales, el activismo de los trabajadores regiomontanos era el resultado de influjos políticos e ideológicos extranjeros que nada tenían que ver con las condiciones materiales de los obreros. Reiteradamente, se presentaron textos donde se advertía la existencia de un proyecto imperialista ruso que no tardaría en llegar a Monterrey. Ante la supuesta pretensión de Lenin y Trotski de enviar "furiosos discípulos" a todas las naciones del mundo para predicar una revolución universal, El Porvenir manifestó su intención de publicar, a partir de febrero de 1919, algunos "estudios" sobre el bolcheviquismo que evidenciaban la amenaza que éste implicaba. ${ }^{17}$

Este trato mediático negaba la existencia de un problema social complejo que debía resolverse con base en una negociación entre patrones y representantes obreros. Tal negación se fundamentaba en la representación que los empresarios tenían sobre sus fuerzas laborales: para ellos, los trabajadores de Monterrey no contaban con la aptitud necesaria para movilizarse por sí mismos.

Es difícil determinar hasta qué punto los empresarios nuevoleoneses realmente creyeron en una posible invasión rusa. ¿Se trataba de

17 El Porvenir, 6 de febrero de 1919.

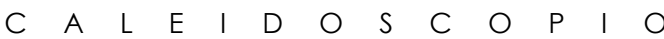


una mera maniobra política que intentaba atemorizar a la sociedad e inducir al rechazo del movimiento obrero? ¿Los patrones se negaban a aceptar las fallas de los mecanismos administrativos y de control dentro de sus fábricas, siendo la invasión bolchevique la única explicación de la conducta crítica de sus trabajadores? Si bien es cierto que el socialismo y el comunismo tuvieron una fuerte presencia en la década de 1910 (el Partido Comunista Mexicano se fundó en 1919) y que a partir de 1917 puede observarse cierto perfil ideológico en los obreros mexicanos (en el uso del lenguaje de clase, por ejemplo), las circunstancias en torno al activismo ruso y el mexicano eran muy diferentes. Particularmente en Monterrey, las exigencias laborales eran específicas: mejorar sus condiciones de vida y garantizar los derechos fundamentados en la Constitución. No obstante, durante décadas (por lo menos hasta finales de los años treinta), El Porvenir utilizó a Rusia para ejemplificar el peligro que suponían las movilizaciones laborales en México.

El diario de Arenales presentaba noticias sobre huelgas y protestas obreras con encabezados que inculcaban miedo, tales como "El pueblo se entrega a un odioso saqueo" o "Los habitantes huyen en completa miseria". ${ }^{18}$ Con este tipo de títulos se buscaba que los regiomontanos asociaran las movilizaciones laborales con la destrucción.

Es pertinente señalar que, envalentonados por el artículo constitucional 123, los trabajadores de la industria acerera efectuaron múltiples huelgas entre 1918 y 1920, las cuales cohesionaron a más de 10 mil operarios de Monterrey y sus zonas aledañas. ${ }^{19}$ Este hecho representó una de las más grandes amenazas para la hegemonía de la élite empresarial, ya que los obreros de uno de los sectores más importantes de Nuevo León estaban decididos a replantear su relación con el orden económico. No es de extrañarse, pues, que El Porvenir estuviese dispuesto a utilizar el miedo como recurso de combate contra la "fiebre constitucionalista". Así, el diario alertaba a los regiomontanos que en Guadalajara los huelguistas atacaban impunemente a los

18 El Porvenir, 2 de marzo de 1919 y 3 de mayo de 1922.

19 En 1918, los trabajadores de la planta metalúrgica ASARCO efectuaron un paro de labores, debido al incumplimiento de la gerencia del artículo constitucional 123. Motivados por este suceso, los obreros de Fundidora Monterrey impulsaron un movimiento de protesta que se generalizó. Rojas Sandoval, Monterrey: poder político..., op . cit., pp. 121-124. 
obreros católicos y agredían "del modo más doloroso" a gente indefensa, inclusive a niños. Asimismo, se afirmaba que en Veracruz los trabajadores comunistas disparaban constantemente armas de fuego e invadían residencias privadas. ${ }^{20}$

La campaña en contra del comunismo y las movilizaciones obreras en México debe interpretarse como un plan diseñado para contrarrestar la radicalización de los trabajadores regiomontanos. Los empresarios concibieron el activismo laboral como una amenaza al sistema económico establecido, la cual debía ser combatida a través de diversos frentes. El Porvenir llegó a proponer la organización de grupos inspirados en el fascismo italiano, que era considerado como una respuesta patriótica ante el avance del comunismo en el mundo. Bajo esta óptica, se publicaban opiniones como la siguiente:

Italia estuvo a punto de hundirse en el mismo abismo en que se ha sepultado Rusia [...]. Los obreros mal aconsejados y afiliados en su mayoría al bolshevismo Leninista llegaron a confiscar numerosas fábricas $[\ldots \mid$ mientras que un gobierno inepto $|\ldots|$ parecía incapaz de conjurar el enorme peligro porque atravesaba la patria. Fue entonces cuando surgió el Fascismo como una reacción contra el espíritu disolvente de las doctrinas bolsheviques. Puede decirse que esa contienda que asumió proporciones de verdadera lucha intestina duró cerca de dos años hasta que al fin el patriotismo se sobrepuso a la traición. ${ }^{21}$

Para El Porvenir, el fascismo italiano era un movimiento admirable cuyo logro más notable fue lograr que numerosos obreros desertaran del sindicalismo y de los centros socialistas para volver "por el buen camino". Esta interpretación explica por qué el diario regiomontano aplaudió que, a finales de 1922, surgiera en Xalapa (Veracruz) una agrupación fascista que se propuso combatir el comunismo. ${ }^{22}$

La estrategia del uso del miedo se reforzó con la difusión de textos que intentaban comprobar científicamente, a través de la explicación del funcionamiento de la psicología colectiva, la incapacidad de las masas para el razonamiento y la organización, en virtud de su "natura-

20 El Porvenir, 3 de mayo de 1922.

21 El Porvenir, 10 de noviembre de 1922.

22 El Porvenir, 21 de noviembre de 1922. 
leza violenta y primitiva", así como de su inherente corruptibilidad. ${ }^{23}$ De este modo, apoyándose en las ideas de Gustavo Le Bon, El Porvenir aseguraba:

El hombre que forma parte de una multitud $[\ldots]$ pierde todo su espíritu crítico, toda su aptitud razonadora; su mentalidad se rebaja hasta la de un primitivo $[\ldots]$. Los caracteres psicológicos comunes que constituyen el alma de las muchedumbres, son: $|\ldots|$ crueldad sin límites, intolerancia excesiva y obediencia servil a sus dirigentes.

A la libertad, las muchedumbres han preferido siempre la igualdad dentro de la servidumbre. Cuando se rompen los frenos sociales que contienen los instintos de las multitudes, caen éstas muy pronto en la barbarie ancestral. ${ }^{24}$

Para el diario de Arenales, ceder una vez ante las peticiones de cualquier movilización obrera implicaba otorgarle consciencia de su fuerza, de modo que si alcanzaba una victoria, por mínima que fuese, no pararía allí y exigiría cada vez más prerrogativas. ${ }^{25}$ Por ello, se construyó una escena teatral maniquea, en donde actuaban invasores y patriotas, constructores y destructores.

A principios de la década de 1920, la veracidad e imparcialidad se plantearon como principios fundamentales del ejercicio periodístico, sin embargo, carecer de ellos no representaba ningún problema ético para El Porvenir. En agosto de 1922, se celebró en la Ciudad de México la Exposición de la Prensa Regional, en la cual se discutieron los valores que debían regir las publicaciones de provincia. A propósito de este evento, el diario regiomontano publicó un artículo que criticó a la prensa capitalina, particularmente por su pretensión de objetividad, y expresó que un periódico, más allá de su labor como informador de los hechos cotidianos, debería buscar incidir en el curso de los acontecimientos:

Creemos que el periódico que tiene una información muy amplia y completa es un buen periódico, pero que es mejor periódico el que tiende en forma apropiada otras secciones en las que se puede hacer labor de más

23 El Porvenir, 31 de enero de 1919.

24 El Porvenir, 11 de febrero de 1919.

25 El Porvenir, 11 de febrero de 1919. 
trascendencia. Se puede informar muy bien y no hacer a la postre obra ninguna y se puede en cambio ser incompleto en la información y hacer bienes positivos a la comunidad sembrando, entre la gente que lee, buenas ideas, sobre todo en estos tiempos en que una de las grandes y graves enfermedades nacionales es la desorientación en que se agitan y mueven las conciencias. ${ }^{26}$

La declaración anterior no debe extrañarse. Desde sus primeros meses de vida, El Porvenir confesó que no pretendía ser un mero informador, sino influir en la dirección y formación de la nación en un momento en el cual el patriotismo debía ser una obligación de todo periodista. ${ }^{27} \mathrm{El}$ Porvenir justificaba así un proyecto ideológico tendente a evitar que las reformas sociales del Estado revolucionario entraran en contradicción con los valores y prácticas del empresariado regiomontano.

Resulta sugestivo que, ante el panorama estratégicamente fomentado por El Porvenir, sus redactores cuestionaran: “¿Qué piensa la clase trabajadora?". ${ }^{28}$ Esta pregunta evidencia uno de los cambios que la Revolución provocó en la prensa de Monterrey, a saber: que la industria periodística prestara atención -con mayor o menor disposición- al sector obrero. Durante el régimen reyista, las fuerzas laborales no fueron contempladas para deliberar en los medios impresos, pero los tiempos revolucionarios revirtieron esta situación. Desde luego que no era intención del diario de Arenales abrir un espacio de confrontación de opiniones diferentes, pero es necesario señalar el interés por generar un público lector compuesto por trabajadores urbanos, porque este hecho evidencia la relevancia de los obreros en la sociedad posrevolucionaria; probablemente, su opinión comenzaba a ser contemplada como un factor decisivo en los asuntos políticos de la época.

El Porvenir declaró que daría a conocer las inquietudes de los trabajadores regiomontanos acerca de "los problemas que agitan al mundo moderno". En consecuencia, divulgó opiniones de algunos obreros afines a las ideas del empresariado. Por ejemplo, el 4 de febrero de 1919, se publicó el siguiente comentario del obrero Dámaso Cantú:

26 El Porvenir, 19 de agosto de 1922.

27 El Porvenir, 27 de marzo de 1919.

28 El Porvenir, 4 de febrero de 1919. 
Los obreros de nuestro país aunque en lo general se han distanciado un tanto de sus deberes y obligaciones como ciudadanos [... | han aventajado algo $[\ldots]$. Pero es un deber de las clases obreras, una obligación forzosa, si queremos que nuestro país sea libre, poderoso y civilizado, ayudar de buena voluntad y desinteresadamente a nuestros gobernantes. ${ }^{29}$

Entre 1919-1922 -por lo menos- se puede observar un gran empeño en difundir la importancia de la armonía entre trabajadores, patrones y gobierno, pero también en generar auténtica simpatía hacia las ideas de los hombres de negocios. Para la élite empresarial, el éxito de esta labor era importante en caso de un eventual enfrentamiento con las organizaciones obreras, así como con las administraciones estatal y federal; por ello, del mismo modo que el Estado y la Iglesia católica, pugnó para establecer su hegemonía sobre las bases populares. ${ }^{30}$

La tarea de suscitar apego a la visión empresarial del mundo no podía alcanzarse únicamente mediante la estrategia del uso del miedo, sin importar lo reiterativo del discurso anticomunista o lo trágico de los numerosos casos presentados. El Porvenir también promovió los supuestos beneficios culturales generados por las fábricas de la élite, a través de escritos que empleaban el lenguaje decimonónico de la teoría racial de la civilización (la cual sostuvo la idea de que existían culturas superiores en el mundo, cuyas características podían ser incorporadas en las razas inferiores para su mejoramiento). Así, por ejemplo, se publicaron textos sobre la Cervecería Cuauhtémoc, en los que se enfatizaba el papel civilizatorio que la prestigiada familia Garza Sada (propietaria de la empresa) desempeñaba en Monterrey. ${ }^{31}$

29 El Porvenir, 4 de febrero de 1919.

30 Knight, Alan, "Estado, revolución y cultura popular en los años treinta", en Águila, Marcos Tonatiuh (coord.), Perspectivas sobre el cardenismo. Ensayos sobre economía, trabajo política y cultura en los años treinta, México, UAM-A, 1996, p. 298. Cabe mencionar que la élite empresarial de Monterrey, en los años treinta, entró en conflicto con el gobierno de Lázaro Cárdenas, a raíz de una huelga efectuada en la vidriera propiedad de la familia Garza Sada. La pugna fue tan fuerte, que el sector empresarial organizó una marcha anticomunista a la cual acudieron -según la prensa- alrededor de 40 mil personas. Dicha movilización se promovió en El Porvenir, además, numerosos ejemplares del diario fueron repartidos gratuitamente dentro de las fábricas de la ciudad. El Porvenir, 4 de febrero de 1936.

31 El Porvenir, 8 de junio de 1922; 28 de octubre de 1922; y 14 de noviembre de 1922. 
Para El Porvenir existían básicamente dos tipos de naciones en el mundo: las civilizadas y las no civilizadas. Una peculiaridad de las primeras era que todos sus estratos sociales, desde los más bajos hasta los más altos, bebían cerveza. ${ }^{32}$ Bajo este razonamiento, el diario regiomontano incitaba a sus lectores al consumo de los productos elaborados en Cervecería Cuauhtémoc. Desde luego que El Porvenir obtuvo ganancias económicas del fomento a la cerveza, porque los textos que presentaba iban acompañados de publicidad (por la cual, evidentemente, cobraba).

Este binomio sintetiza el discurso propagado por El Porvenir para contrarrestar la influencia de los trabajadores organizados: activismo obrero como sinónimo de barbarie e industria como elemento civilizatorio. Conviene señalar que, a principios del siglo xx, la prensa sensacionalista europea difundió ampliamente una supuesta confrontación entre el mundo civilizado y el salvajismo, con la intención de legitimar el expansionismo (acordado en la Conferencia de Berlín, 1884-1885) de las potencias occidentales en África. ${ }^{33}$ De acuerdo con Tenorio Trillo, en esos años se asoció lo industrial y capitalista con el camino ideal que todas las naciones del mundo debían seguir para alcanzar la modernidad; ${ }^{34}$ este imaginario se arraigó en Monterrey durante el régimen de Bernardo Reyes y fue propagado por El Porvenir tras el estallido de la Revolución.

Como emisario de los influyentes empresarios de Monterrey, el diario fundado por Arenales se encargó de construir, durante una generación (1919-1950), representaciones colectivas fundamentadas en el orgullo industrial y el miedo hacia las movilizaciones obreras. En este sentido, El Porvenir de la primera mitad del siglo Xx debe entenderse como un instrumento de penetración ideológica, cuya historia tiene mucho que decir acerca de la experiencia y la reacción de la élite empresarial ante los cambios provocados por la Revolución.

32 El Porvenir, 18 de abril de 1920.

33 En dicha Conferencia, doce naciones europeas (a las que se incluyeron Estados Unidos y Turquía) se reunieron para impulsar la exploración y ocupación de África. Este expansionismo se justificó en nombre del progreso. Bertaux, Pierre, África, desde la prehistoria hasta los Estados actuales, México, Siglo XXI Editores, 1995, pp. 167, 170 y 188.

34 Tenorio Trillo, Mauricio, Artilugio de la nación moderna. México en las exposiciones universales, 1880-1930, México, Fondo de Cultura Económica, 1998, pp. 10 y 14. 


\section{CONCLUSIONES}

En el artículo se evidenció cómo El Porvenir fue un proyecto que surgió para contrarrestar la influencia del movimiento obrero regiomontano, labor llevada a cabo principalmente mediante el uso estratégico del miedo. Es decir, que el diario fundado por Arenales surgió para cumplir un papel político específico. Pero también desempeñó una función cultural, pues buscó construir un imaginario colectivo acorde a la visión del mundo de la élite empresarial.

Puede decirse que la Revolución provocó que en Monterrey emergiera una prensa informativa-combativa, que se preocupó por intervenir en el curso de los acontecimientos. En este sentido, El Porvenir debe ser entendido y analizado como un actor político más de su tiempo; propagar cierto tipo de noticias (o no hacerlo) lo convirtió en una pieza crucial para la defensa de los grandes intereses empresariales. La historia de la prensa regiomontana constituye así un campo de estudio que contribuye a entender, de manera más profunda, las confrontaciones entre obreros y patrones, así como la resistencia de estos últimos a las reformas planteadas por el Estado revolucionario.

¿Qué grado de influencia tuvo El Porvenir? Ésta es una pregunta no resuelta en el artículo, pues para responderla era necesario realizar un estudio sobre la recepción del diario. La investigación se centró en examinar el papel que desempeñó El Porvenir en una coyuntura específica, la repercusión de su función merece ser analizada profundamente en un periodo mucho más amplio, ya que el ritmo de cambio de los imaginarios colectivos es lento. Aun así, no debe menospreciarse el poder de penetración de la prensa empresarial.

Lo cierto es que, a través de El Porvenir, puede advertirse la conformación de un empresariado politizado que vio en la prensa un importante instrumento de combate capaz de hacer frente al activismo obrero. Otro tema pendiente en este artículo fue la respuesta de los trabajadores ante los discursos difundidos por el diario de Arenales (punto relevante a tratar si se considera que los lectores no son receptores pasivos de los mensajes). Un trabajo que atienda a tal tema sería de utilidad para comprender qué tan dinámica y amplia fue la esfera pública regiomontana.

A pesar de los vacíos antes mencionados, esta investigación aporta conocimiento valioso sobre la prensa empresarial contemporánea de Monterrey, un aspecto poco explorado por los historiadores locales. 
Asimismo, permite hacer estudios comparativos que a su vez pueden contribuir a elaborar interpretaciones generales del periodo mucho más completas.

FUENTES CONSULTADAS

\section{Archivos}

Archivo Histórico de Nuevo León.

\section{Hemerografía}

El Porvenir.

\section{Bibliografía}

Bertaux, Pierre, África, desde la prehistoria hasta los Estados actuales, México, Siglo XXI Editores, 1995.

Burkholder de la Rosa, Arno, "El periódico que llegó a la vida nacional. Los primeros años del diario Excélsior (1916-1932)", Historia Mexicana, México, El Colegio de México, vol. LVIII, núm. 4, abril-junio de 2009, pp. 1371-1418.

Cavazos Garza, Israel, Escritores de Nuevo León, diccionario biobibliográfico, Monterrey, Universidad Autónoma de Nuevo León, 1996.

Cerutti, Mario, "Producción capitalista y articulación del empresariado en Monterrey (1890-1910)", en Labastida, Julio, (comp.), Grupos económicos y organizaciones empresariales en México, México, Biblioteca Iberoamericana-Alianza Editorial Mexicana-Universidad Nacional Autónoma de México, 1986, pp. 15-55.

García Aguilar, Eduardo (investigación, selección y prólogo), Porfirio Barba Jacob. Escritos mexicanos, Bogotá, Fondo de Cultura Económica, 2009.

Knight, Alan, "Estado, revolución y cultura popular en los años treinta", en Águila, Marcos Tonatiuh (coord.), Perspectivas sobre el cardenismo. Ensayos sobre economía, trabajo, política y cultura en los años treinta, México, UAM-A, 1996, pp. 297-324.

Nuncio, Abraham, Visión de Monterrey, México, Fondo de Cultura Económica, 2006. 
Rojas Sandoval, Javier, Monterrey: poder político, obreros y empresarios en la coyuntura revolucionaria, Monterrey, Facultad de Filosofía y Letras, Universidad Autónoma de Nuevo León, 1992.

Movimiento obrero y partidos políticos en Nuevo León. 1910-1920, Monterrey, OIDMO, A. C., 1982.

Rousset Banda, Guillermo (coord.), Porfirio Barba Jacob, obra poética, México, Editorial Domés, 1985.

Snodgrass, Michael, Deferencia y desafío en Monterrey. Trabajadores, paternalismo y Revolución en México, 1890-1950, Monterrey, Fondo Editorial de Nuevo León, 2008.

Tenorio Trillo, Mauricio, Artilugio de la nación moderna. México en las exposiciones universales, 1880-1930, México, Fondo de Cultura Económica, 1998. 